Check for updates

Cite this: RSC Adv., 2018, 8, 13664

Received 10th February 2018

Accepted 21st March 2018

DOI: $10.1039 / \mathrm{c} 8 \mathrm{ra01306c}$

rsc.li/rsc-advances

\section{Water-soluble and adjustable fluorescence copolymers containing a hydrochromic dye: synthesis, characterization and properties $\dagger$}

\author{
Le Ju, ${ }^{\text {ab }}$ Tianyou Qin, ${ }^{a}$ Ting Zhang, ${ }^{c}$ Peng Wang, ${ }^{a}$ Lan Sheng (DD *a and Sean Xiao-An \\ Zhang (D) bc
}

Water solubility and adjustable fluorescence properties have been successfully implemented in the hydrochromic amino rhodamine via copolymerization. Four copolymers have been synthesized and clearly characterized by UV-Vis spectroscopy, proving greater detail than the commonly used NMR and IR technologies. The four copolymers have good solubility in pure water and in many common organic solvents, while preserving the hydrochromism of the dye monomer. Based on aggregation and dispersion of the copolymers as adjusted by solvent media and temperature, reversible fluorescence properties were successfully realized. Furthermore, their luminescence in solid state was observed. These studies are of great significance for expanding the application of hydrochromic dyes in biological fields and promoting green industrialization.

\section{Introduction}

As a source of life, water participates in almost all metabolic processes, supplies solvents to cells and organelles, serves as a lubricant of the organism, and is the foundation for continuance of life. ${ }^{1}$ Inspired from nature and life, scientists are actively exploring excellent water-soluble drugs or materials with the hope of favorable biological applications or green industrial production to better serve human health and life..$^{2-7}$ Recently, hydrochromic materials have received much concern due to their wide application in rewritable imaging on paper, ${ }^{8,9}$ humidity sensors, ${ }^{10-14}$ anti-counterfeit, ${ }^{15}$ encrypting information, ${ }^{16,17}$ etc. To meet the green industrial demand and to further extend their applications, water-soluble hydrochromic materials with fluorescence are very necessary but rare.

Rhodamine compounds are widely used for dyes ${ }^{18,19}$ and ion detections ${ }^{20-22}$ due to their high molar extinction coefficients, excellent photostability, good fluorescent property with high quantum yields and relative ease of modification. In our previous study, it was found that modifying rhodamine with amino $\left(\mathbf{N H}_{2}-\mathbf{R h}\right)$ can endow it with hydrochromism for application in water-jet rewritable paper. ${ }^{23}$ That is, isomerization of

${ }^{a}$ College of Chemistry, Jilin University, Changchun, 130012, China. E-mail: shenglan17@jlu.edu.cn

${ }^{b}$ Department of Chemistry and Pharmacy, Zhuhai College of Jilin University, Zhuhai, 519041, China

'State Key Lab of Supramolecular Structure and Materials, College of Chemistry, Jilin University, Changchun, 130012, China

$\dagger$ Electronic supplementary information (ESI) available. See DOI: $10.1039 / \mathrm{c} 8 \mathrm{ra} 01306 \mathrm{c}$ the lactone form of $\mathbf{N H}_{2}-\mathbf{R h}$ takes place to an open-ring form in the presence of water, showing a bright colour. Conversely, when water disappears, the open-ring structure reversibly returns to its lactone form and the corresponding colour fades simultaneously. However, after modifying with an amino group, the fluorescence of rhodamine was weakened greatly after the addition of water, especially in solid or solid substrate, showing almost no fluorescence. ${ }^{23}$ In order to expand its applications, it is necessary to restore its fluorescence properties, especially in water or in solid state.

Poly( $N$-isopropylacrylamide) (PNIPAM), as a water-soluble and thermosensitive smart polymer, has been applied to drug delivery, ${ }^{24-26}$ controlled-release carriers, ${ }^{27}$ microreactor template synthesis of nanoparticles, ${ }^{28,29}$ and optical materials. ${ }^{30-32}$ It is water soluble due to the strong hydrogen-bonding interaction between $N$-isopropylacrylamide groups and water molecules. When the temperature is higher than its "lower critical solution temperature" (LCST), ${ }^{33-36}$ its solubility is greatly reduced. This thermo-controlled solubility property is reversible and has given PNIPAM a wide range of applications. Fluorescence tags have been copolymerised with PNIPAM $^{37-39}$ and used to elucidate polymer properties; ${ }^{40,41}$ however, the obtained fluorescence response signals are often weak. Thus, copolymers with PNIPAM and high-intensity fluorophores are still being pursued.

In the present work, we have synthesized four copolymers, $\operatorname{poly}\left(\mathbf{A M}^{-R h_{x}}\right.$ co $\left.\mathbf{N I P A M}_{y}\right) \mathrm{s}$, with different ratios of $x$ to $y$, by grafting simply modified $\mathbf{N H}_{\mathbf{2}}$ - $\mathbf{R h}$ unit-AM-Rh to water-soluble PNIPAM chains by copolymerization. It is expected that the poly(AM-Rh ${ }_{x}$ co NIPAM $_{y}$ )s will maintain the hydrochromic property of $\mathbf{N H}_{\mathbf{2}}-\mathbf{R h}$ while gaining water solubility and fluorescence. 


\section{Results and discussion}

Synthesis and characterization of poly $\left(\mathrm{AM}-\mathrm{Rh}_{x}\right.$ co $\left.\mathrm{NIPAM}_{y}\right)$

The general synthesis route of the poly $\left(\mathbf{A M}-\mathbf{R h}_{\boldsymbol{x}}\right.$ co $\left.\mathbf{N I P A M}_{y}\right)$ copolymers is summarized in Scheme 1. AM-Rh was obtained by acylation reaction of $\mathbf{N H}_{2}-\mathbf{R h}$ and acryloyl chloride, with $52 \%$ yield, and characterized by NMR and MS analysis (see Fig. S1 $\dagger$ ). Poly(AM-Rh $\boldsymbol{A}_{\boldsymbol{x}}$ co NIPAM $_{\boldsymbol{y}}$ )s were successfully synthesized by radical polymerization of NIPAM monomer with AM-Rh as pink solids, which were obtained by precipitation from methanol and diethyl ether. The solids were further purified through dialysis (using a membrane with molecular weight cutoff of 5000 ) in methanol for $60 \mathrm{~h}$ to remove the small residues of AMRh and NIPAM. Poly(AM-Rh co NIPAM $_{y}$ )s with different ratios $(x / y)$ of the respective units could be regulated by varying the reaction molar ratio of AM-Rh to NIPAM for polymerization. Four copolymers, P1/50, P1/100, P1/200, and P1/345, were prepared with AM-Rh : NIPAM molar ratios of $1: 50,1: 100$, $1: 200$, and $1: 345$, respectively. In addition, as a control experiment, poly(NIPAM) (P0) was also prepared.

Characterization of polymers containing functional molecules has always been a difficult problem. NMR and IR technologies are currently used commonly; however, the information indicating bonding between polymers and functional molecules is not clear from these characterizations most of the time (Fig. S $2 \dagger$ ), especially the ratio of copolymers to functional molecules. ${ }^{\mathbf{4 2}}$ For example, signals of poly(AM-Rh $\mathbf{x}_{\boldsymbol{x}}$ co NIPAM $_{y}$ ) in NMR spectra generate a broad peak, mainly belonging to $\mathrm{P} 0$, which is insufficient to identify information for AM-Rh (Fig. S3 $\dagger$ ). Considering that both poly(AM-Rh $\mathbf{x}_{\boldsymbol{x}}$ co $\mathbf{N I P A M}_{\boldsymbol{y}}$ ) and AM-Rh have obvious colour characteristic absorption peaks, the characterization of $\operatorname{poly}\left(\mathbf{A M}-\mathbf{R h}_{\boldsymbol{x}}\right.$ co NIPAM $_{y}$ ) was attempted by UV-Vis spectroscopy. Results show that the maximum absorption peaks $\left(\lambda_{\max }\right)$ for the four copolymers are nearly the same at $539 \mathrm{~nm}$. They distinctly show $\lambda_{\max }$ of the mixture of P0 and AM-Rh centred at $542 \mathrm{~nm}$, which is the same with the $\lambda_{\max }$ of monomer AM-Rh (Fig. 1a). This result indicates that $\mathbf{A M - R h}$ is successfully covalently grafted onto the polymer chains. It also verifies that spectroscopy is an effective

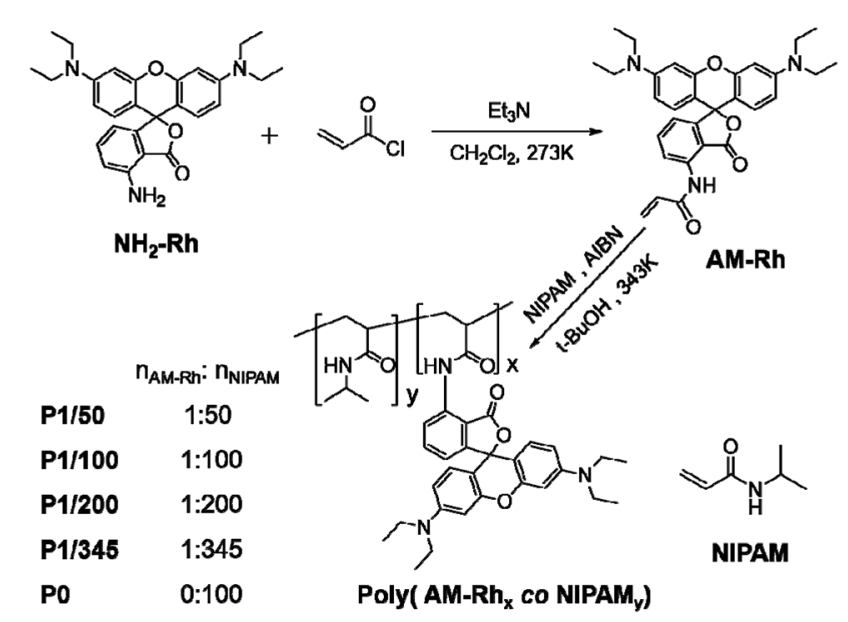

Scheme 1 Synthesis of AM-Rh and poly $\left(A M-R h_{x}\right.$ co NIPAM $)$

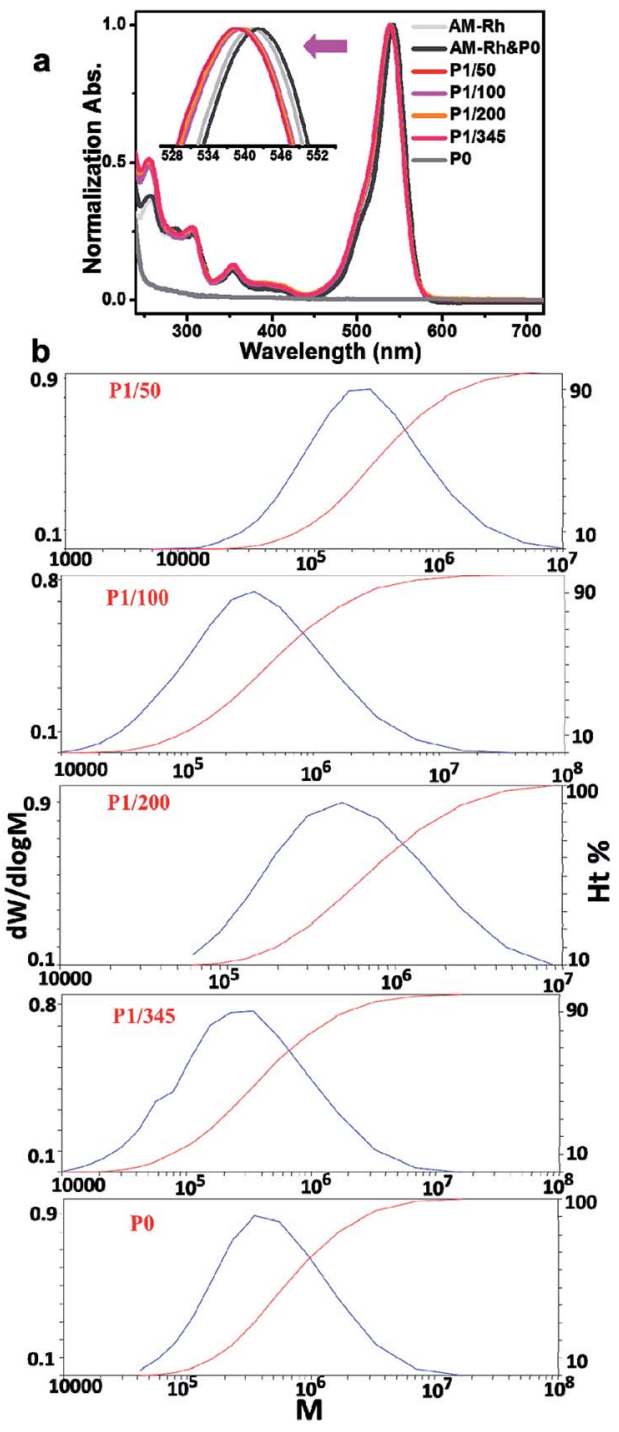

Fig. 1 (a) Normalized UV-Vis spectra of P1/50, P1/100, P1/200, P1/ 345, PO, AM-Rh and mixture of AM-Rh and PO (AM-Rh \& PO) in methanol. (b) The plots of dwdlog $M v s$. M for poly $\left(A M-R_{x}\right.$ co NIPAM $)$ $\mathrm{s}$ and $\mathrm{PO}$

way to characterise polymers containing units with characteristic absorption.

The ratio $(x / y)$ of respective units of poly(AM-Rh $x$ co NIPAM $\left._{y}\right)$ was therefore determined by combining UV-Vis spectroscopy and gel-permeation chromatography (GPC). The test method and processes are illustrated in detail in Fig. S4. $\dagger$ Firstly, the molar absorption coefficients $(\varepsilon)$ of poly $\left(\mathbf{A M}-\mathbf{R h}_{\boldsymbol{x}} \boldsymbol{c o} \mathbf{N I P A M}_{\boldsymbol{y}}\right) \mathrm{s}$ in open-ring form in methanol were estimated. Hereon, $\varepsilon$ values for $\operatorname{poly}\left(\mathbf{A M}-\mathbf{R h}_{\boldsymbol{x}}\right.$ co $\left.\mathbf{N I P A M}_{\boldsymbol{y}}\right) \mathrm{s}$ are approximately considered to be the same as the AM-Rh's $\varepsilon$, which was calculated to be $111850 \mathrm{~L} \mathrm{~mol}^{-1} \mathrm{~cm}^{-1}$ by standard curve method according to Beer-Lambert law (Fig. S5 $\dagger$ ). Secondly, the number-averaged molecular weight $\left(M_{\mathrm{n}}\right)$ of the poly $\left(\mathbf{A M}-\mathbf{R h}_{\boldsymbol{x}}\right.$ co $\left.\mathbf{N I P A M}_{\boldsymbol{y}}\right) \mathrm{s}$ was measured by gel permeation chromatography (GPC) (Fig. 1b and $56 \dagger)$. On the basis of $M_{\mathrm{n}}$ of poly(AM-Rh $\mathbf{R}_{\boldsymbol{x}}$ co $\left.\mathbf{N I P A M}_{y}\right)$, methanol solutions of $\operatorname{poly}\left(\mathbf{A M}-\mathbf{R h}_{\boldsymbol{x}}\right.$ co $\left.\mathbf{N I P A M}_{\boldsymbol{y}}\right)$ with a certain concentration $\left(C_{\text {poly(AM-Rhx }}\right.$ co NIPAMy) $)$ were obtained, and the 
Table 1 Composition of the poly(AM-Rh $h_{x}$ co NIPAMy)

\begin{tabular}{lllllll}
\hline Polymer & $n_{\text {AM-Rh }}: n_{\text {NIPAM }}$ & $M_{\mathrm{n}}$ & $D$ & $x$ & $y$ & $x / y$ \\
\hline P0 & $0: 100$ & 332480 & 1.46 & 0 & - & - \\
P1/50 & $1: 50$ & 148295 & 2.19 & 17 & 1213 & $1 / 71$ \\
P1/100 & $1: 100$ & 183190 & 1.26 & 11 & 1571 & $1 / 142$ \\
P1/200 & $1: 200$ & 395317 & 1.74 & 15 & 3430 & $1 / 229$ \\
P1/345 & $1: 345$ & 160250 & 2.17 & 4 & 1373 & $1 / 343$ \\
\hline
\end{tabular}

absorbance $\left(A_{\text {poly(AM-Rhx }}\right.$ co NIPAMy) $)$ of the open-ring form of grafted AM-Rh was determined by UV-Vis spectroscopy. According to Beer-Lambert law and $\varepsilon$, the concentrations of grafted AM-Rh on poly(AM-Rh $x$ co NIPAM $\left._{y}\right)\left(C_{\mathbf{A M}-\mathrm{Rh}}\right.$ grafted on polymer) were calculated, respectively. Then, the value of $x$ was obtained from $C_{\mathbf{A M}-\mathrm{Rh} \text { grafted on polymer }} / C_{\mathbf{p o l y}(\mathbf{A M} \text {-Rh } x \text { co NIPAMy) }}$, and the value of $y$ was obtained from $M_{\mathrm{nAM}-\mathrm{Rh}} x+M_{\mathrm{nNIPAM}} y=M_{\mathrm{n}}{ }^{-}$ poly(AM-Rh $x$ co NIPAMy). Finally, the value of $x / y$ for poly(AM-Rh $x$ co NIPAM $_{y}$ ) was obtained as well (Table 1).

\section{Water solubility and hydrochromic property}

Solubility, especially water solubility, is of great significance in extending the application of materials in biological fields or industry. Therefore, water solubility of the prepared copolymers was firstly investigated. As expected, the four copolymers all have good water solubility. As an example, P1/100 can dissolve completely in water, at a solubility of more than $20 \mathrm{mg} \mathrm{mL}^{-1}$. In addition, it also has good solubility in most organic solvents, such as chloroform, methanol, ethanol, tertiary butanol, $\mathrm{N}, \mathrm{N}$ dimethyl formamide (DMF), tetrahydrofuran, dimethyl sulfoxide, acetonitrile, and acetone. As can be seen in Fig. 2a, P1/ 100 was dissolved in water, giving a magenta solution, and the main absorption band for the aqueous solution is between 450-600 nm, which is similar to the maximum absorption of AM-Rh in halochromism (Fig. S7†). The only difference is that the intensity of the shoulder peak around $524 \mathrm{~nm}$ for P1/100 is much higher (Fig. S8†). P1/100 dissolved in DMF solution is colourless, and the main absorption band is less than $350 \mathrm{~nm}$. These results indicate that $\mathrm{P} 1 / 100$ exists in lactone form in DMF, while it exists in open-ring form in water. That is, in addition to water solubility, P1/100 maintains its hydrochromic property.

In order to investigate the hydrochromic property in detail, the colour change and corresponding UV-Vis spectra of P1/100 in the DMF solution with increasing water content were examined. As shown in Fig. 2b, the colourless DMF solution of P1/100 gradually changed to magenta with the increase of water content, and the absorption peak intensity at around $550 \mathrm{~nm}$, assigned to the main absorption of its open-ring form, also gradually increased with the increase of water content until it reached $70 \%$. With further increase in water content $(80 \%$ and $90 \%$ ), the absorption intensity at around $550 \mathrm{~nm}$ decreased, accompanied by the increase of absorption intensity for the shoulder peak at around $524 \mathrm{~nm}$. Combined with the absorption spectra of $\mathrm{P} 1 / 100$ in pure water, it can be concluded that the shoulder peak is caused by hydration of the open-ring form of
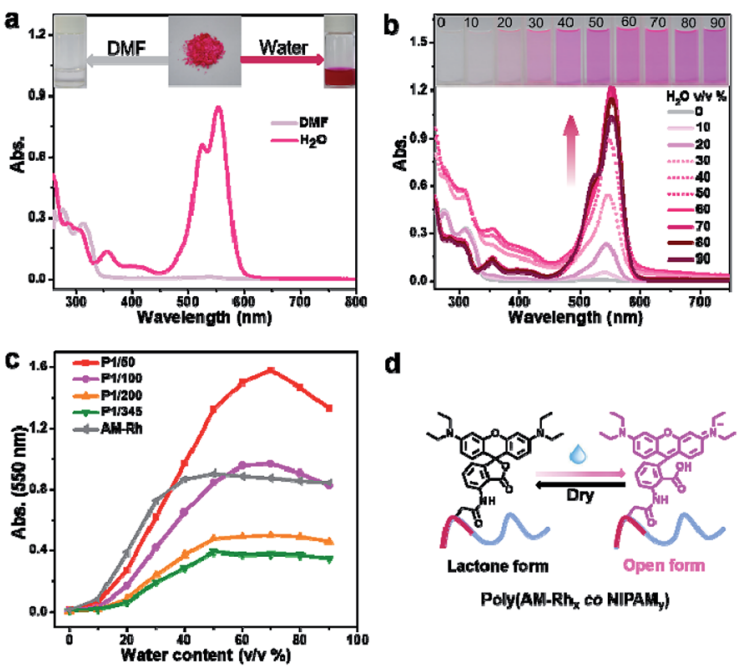

d

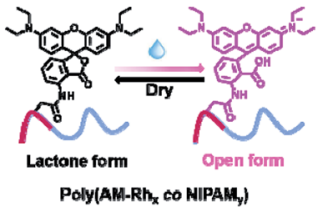

Fig. 2 (a) UV-Vis spectra of $\mathrm{P} 1 / 100$ in water $\left(0.2 \mathrm{mg} \mathrm{mL}^{-1}\right)$ and DMF $\left(0.2 \mathrm{mg} \mathrm{mL}^{-1}\right)$, respectively. Inset: photos of P1/100 in water and DMF. (b) UV-Vis absorption spectra of P1/100 in variable mixtures of DMF and water with increasing percentage of water by volume from 0 to $90 \%\left(0.2 \mathrm{mg} \mathrm{mL}^{-1}\right.$ ). Inset: photographs of the solution of P1/100 in variable mixtures of DMF and water with different amounts of water (from $0 \%$ to $90 \%$ ). (c) Summarized water-content-dependent changes of P1/50, P1/100, P1/200, P1/345 ( C = $1 \times 10^{-5} \mathrm{~mol} \mathrm{~L}^{-1}$ ) AM-Rh for the absorption at $\lambda_{\max }$. (d) Illustration of reversible water-stimulated isomerization between the lactone form and open form of poly(AM$\mathrm{Rh}_{x}$ co NIPAM $)_{y}$.

AM-Rh. ${ }^{23}$ It is also worth mentioning that no isosbestic point was observed for these UV-Vis spectra, which is different from our previously reported hydrochromic dyes. ${ }^{8,23}$ This should be caused by irregular absorption changes of the polymer with varying compositions of the mixed solvents, especially in the binary solvents with water content of $30 \%, 40 \%$ and $50 \%$, in which the copolymer has poor solubility and showed increasing absorption around $250-500 \mathrm{~nm}$. To prove this point, absorption spectra of P0 under the same condition was detected. The obvious poor solubility was also clearly observed in the binary solvents with water content of $30 \%, 40 \%$ and $50 \%$ both in UVVis spectra and pictures (Fig. S9 and S10†). Moreover, the spectra for P1/100 in pure DMF with gradual addition of acid and for AM-Rh in DMF- $\mathrm{H}_{2} \mathrm{O}$ binary solvents with varying water content all have isosbestic points (Fig. S11 and S12†), which further demonstrates our point.

In order to further investigate whether the ratio of dye molecules grafted to the polymer has an effect on their hydrochromism, the three other copolymers of poly(AM- $\mathbf{R} \mathbf{h}_{x}$ co NIPAM $_{y}$ ), that is, P1/50, P1/200 and P1/345, were also tested under the same conditions and compared to P1/100 and AM-Rh. From the curves of their absorbance at $550 \mathrm{~nm}$ with changing water content, we can observe that the four copolymers grafted with AM-Rh all retained their hydrochromic property, while their hydrochromic sensitivity (reflected by slope of the curve changes) decreased, compared to the monomer AM-Rh. In addition, among the four copolymers, as the dye graft rate decreases, their hydrochromic sensitivity decreases, that is, P1/ $50>\mathrm{P} 1 / 100>\mathrm{P} 1 / 200>\mathrm{P} 1 / 345$ (Fig. 2c). Furthermore, the 
maximum absorption intensity for their hydrochromism can also be adjusted by changing the grafting ratio of $\mathbf{A M - R h}$ to NIPAM. That is, the maximum absorption intensity increases gradually with grafting ratio of AM-Rh to NIPAM. Structural change of the dye molecule-grafted poly(AM-Rh ${ }_{x}$ co NIPAM $\left._{y}\right)$ corresponding to hydrochromism, is shown in Fig. 2d.

\section{The fluorescence property of Poly(AM-Rh $x$ co $\left.\operatorname{NIPAM}_{y}\right)$}

Fluorescence properties of poly(AM-Rh $x$ co NIPAM $\left._{y}\right) \mathrm{s}_{\text {were also }}$ investigated. Hereon, P1/100 is also described as an example.

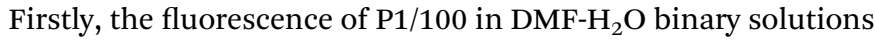
with varying water content was studied. As shown in Fig. 3a, both the intensity and maximum emission peak of the fluorescence spectra for $\mathrm{P} 1 / 100$ have changed with increasing percentage of water. The generation of fluorescence mainly comes from the ring opening of the lactone ring of $\mathbf{A M}-\mathbf{R h}$ to form a conjugation-extended open-ring structure (Fig. S13†). The fluorescence signals for both poly(AM-Rh $\left.\boldsymbol{A n}_{\boldsymbol{x}} \boldsymbol{c o ~ N I P A M}_{\boldsymbol{y}}\right) \mathrm{s}$ and AM-Rh are stronger than $\mathbf{N H}_{\mathbf{2}}-\mathbf{R h}$ under the same conditions (Fig. S14†). The emission peak position gradually red shifted
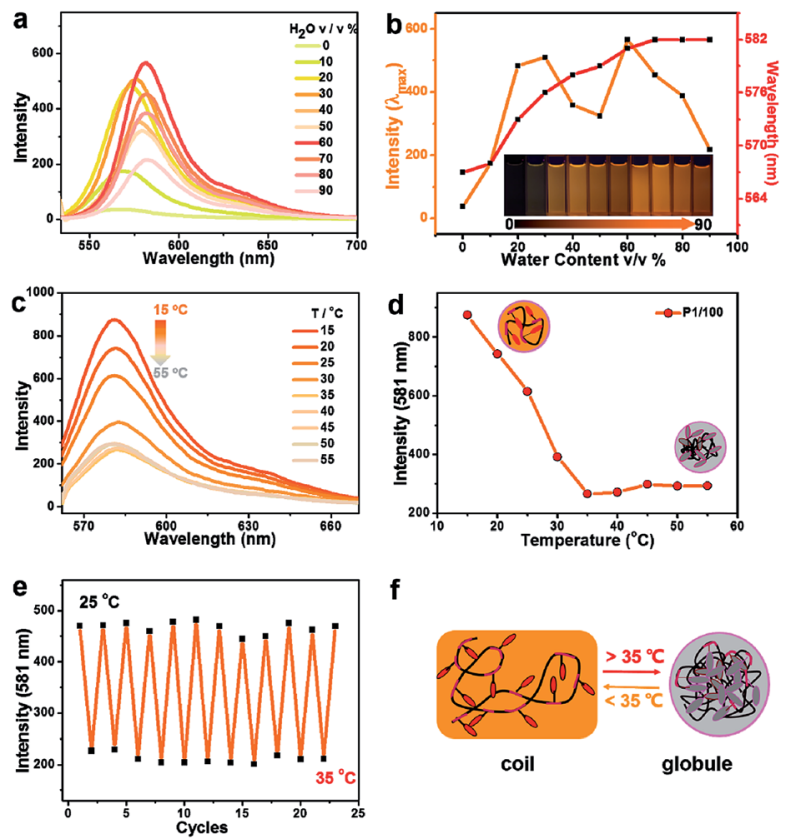

$\mathbf{f}$

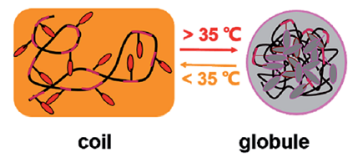

Fig. 3 (a) Fluorescence spectra of P1/100 in variable mixtures of DMF and water, with increasing percentage of water by volume from 0 to $90 \%\left(C=1 \times 10^{-5} \mathrm{~mol} \mathrm{~L}^{-1}, \lambda_{\mathrm{ex}}=530 \mathrm{~nm}\right.$; slit width: $\left.3,1.5,25^{\circ} \mathrm{C}\right)$. (b) Plot of fluorescence intensity at $\lambda_{\max }$ with water content and corresponding maximum emission wavelength $\left(\lambda_{\mathrm{ex}}=530 \mathrm{~nm}\right.$; slit width: 3 , $\left.1.5 ; 25^{\circ} \mathrm{C}\right)$. Inset: Photographs of the solution of P1/100 (0.2 $\left.\mathrm{mg} \mathrm{mL}^{-1}\right)$ in variable mixtures of DMF and water with different amounts of water, from $0 \%$ to $90 \%$. (c) Fluorescence spectra of P1/100 $\left(0.2 \mathrm{mg} \mathrm{mL}^{-1}\right)$ in water with varying temperature from $15{ }^{\circ} \mathrm{C}$ to $55^{\circ} \mathrm{C}$. (d) Temperaturedependent change in fluorescence $\left(\lambda_{\mathrm{ex}}=550 \mathrm{~nm}\right.$; slit width: $\left.1.5,3\right)$ of $\mathrm{P} 1 / 100\left(0.2 \mathrm{mg} \mathrm{mL}^{-1}\right)$ in water, with temperature varied from $15^{\circ} \mathrm{C}$ to $55^{\circ} \mathrm{C}$. (e) A plot of the fluorescence at $581 \mathrm{~nm}$ versus the number of cycles as P1/100 is cycled through heating and cooling between $25^{\circ} \mathrm{C}$ to $35^{\circ} \mathrm{C}\left(\lambda_{\text {ex }}=550 \mathrm{~nm}\right.$; slit width: $\left.1.5,3\right)$. (f) Schematic for fluorescence intensity variation of poly $\left(\mathrm{AM}-\mathrm{Rh}_{x}\right.$ co NIPAM $)$ in water between $25^{\circ} \mathrm{C}$ to $35^{\circ} \mathrm{C}$. with the increase of water content (Fig. $3 \mathrm{~b}$, red line). This is due to the solvation effect on the zwitterionic open-ring form of AM$\mathbf{R h}$, whose ground state dipole moment is less than its excited state dipole moment $\left(\mu_{\mathrm{g}}<\mu_{\mathrm{e}}\right)^{23,43}$

The cause of fluorescence in poly(AM-Rh $\mathrm{A}_{x}$ co $\left.\mathrm{NIPAM}_{y}\right) \mathrm{s}$ was further proved by the fact that similar changes of peak position were observed in AM-Rh under the same conditions (Fig. S15†). Meanwhile, the change trend of P1/100 with increased water content presents an $\mathrm{M}$-shaped (Fig. 3b, orange line). From 0$30 \%$, the fluorescence intensity increases with increasing water content, which is mainly due to the gradual increase of ratio for the open-ring form of $\mathbf{A M}-\mathbf{R h}$; at $30-50 \%$, the intensity decreased gradually, which may be mainly due to the reduction of solubility for $\mathrm{P} 1 / 100$ in the binary solutions as we discussed above; from $60 \%-90 \%$, the intensity decreased again, despite increasing at $60 \%$ due to decrease of absorbance for the openring form of AM-Rh, as shown in Fig. 2c (70-90\%).

Given that poly( $N$-isopropyl acrylamide) is a water-soluble thermo-responsive polymer that undergoes temperatureregulated, reversible coil-to-globule phase transition, the capability of poly(AM-Rh $\boldsymbol{x}_{\boldsymbol{x}}$ co $\left.\mathbf{N I P A M}_{\boldsymbol{y}}\right)$ to achieve tunable fluorescence with temperature variation in water was verified next. Fluorescence spectra change of $\mathrm{P} 1 / 100$ in water was measured by varying the temperature range from $15{ }^{\circ} \mathrm{C}$ to $55^{\circ} \mathrm{C}$ (Fig. 3c). $\mathrm{P} 1 / 100$ is soluble in water (coil), with distinct fluorescence at low temperature $\left(15{ }^{\circ} \mathrm{C}\right)$, but a rise in temperature leads to a gradual fluorescence quenching (Fig. 3d). When temperature is up to $35{ }^{\circ} \mathrm{C}$, the fluorescence intensity reached the lowest, remaining the same as the temperature continued to increase (Fig. 3d). This is presumably due to the formation of copolymer particles ${ }^{44,45}$ associated with the aggregation of copolymer chain (globule) at temperatures exceeding $35{ }^{\circ} \mathrm{C}$. In addition, the decrease in fluorescence intensity due to intermolecular interaction enhanced by increasing temperature is one of the reasons (Fig. S14 $\dagger$ ). Furthermore, this tunable fluorescence intensity change for P1/100, adjusted by increasing and reducing temperature, is reversible and repeatable. As shown in Fig. 3e, nearly no intensity decrease was observed after ten consecutive heating-cooling cycles. The corresponding schematic diagram for fluorescence intensity and state changes of the copolymer chain between the coil form at low temperature $\left(<35{ }^{\circ} \mathrm{C}\right)$ and globule form at high temperature $\left(>35{ }^{\circ} \mathrm{C}\right)$ is illustrated in Fig. 3f.

Fluorescence properties of the four poly(AM-Rh ${ }_{x} \operatorname{co}$ NIPAM $\left._{y}\right)$ $\mathrm{s}$ in solid were also studied. These four polymers have fluorescence emission under solid powder; nevertheless, there is nearly no emission observed for powders of $\mathbf{A M}-\mathbf{R h}$ or $\mathbf{N H}_{\mathbf{2}}-\mathbf{R h}$ (Fig. 4a). The fluorescence emission fluorescence comes from the open-ring form of AM-Rh in the poly(AM-Rh $\left.\boldsymbol{x}_{\boldsymbol{x}} \boldsymbol{c o} \mathbf{N I P A M}_{\boldsymbol{y}}\right)$, which is verified by the powder colour of magenta and the main absorption peak around 500-600 nm via UV-Vis spectroscopy in Fig. 4c. Their fluorescence intensities were qualitatively measured (spectra shown in Fig. 4d) and quantitatively tested by quantum yields (QYs). QYs for the powders of P1/345, P1/200, $\mathrm{P} 1 / 100, \mathrm{P} 1 / 50$, AM-Rh, and $\mathbf{N H}_{\mathbf{2}}-\mathbf{R h}$ are $47.87 \%, 30.45 \%$, $11.16 \%, 5.37 \%, 1.31 \%$, and $0.27 \%$, respectively, as shown in 


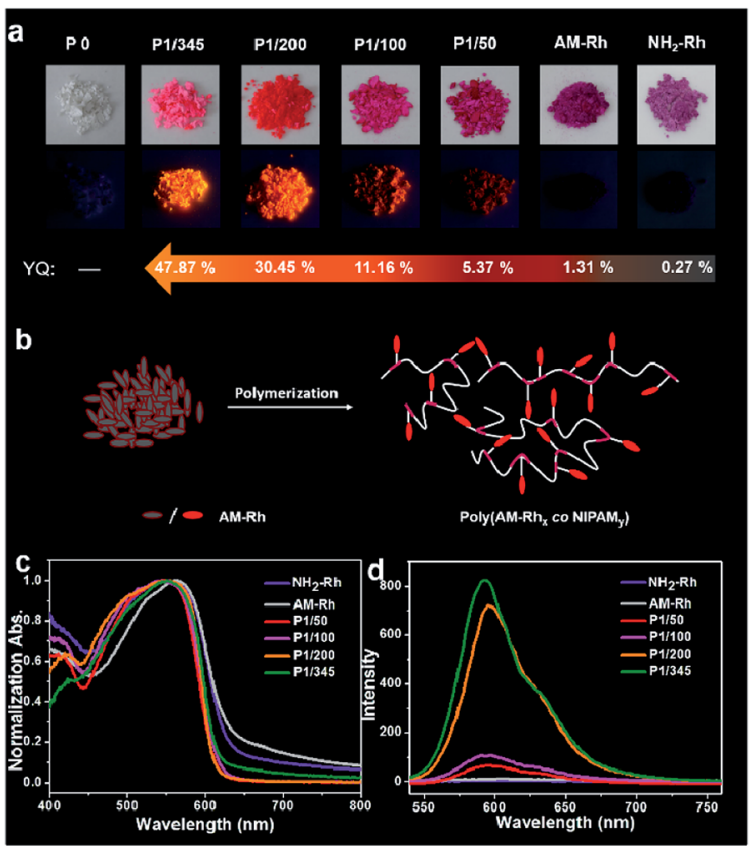

Fig. 4 (a) Photos of the powders of P0, P1/345, P1/200, P1/100, P1/50, $\mathrm{AM}-\mathrm{Rh}$, and $\mathrm{NH}_{2}-\mathrm{Rh}$ under room light and UV light of $365 \mathrm{~nm}$, respectively, and their corresponding quantum yields (QYs). (b) Schematic for the fluorescence emission of AM-Rh- and AM-Rhgrafted poly(AM-Rh co NIPAM $_{y}$ ) in powder form. (c) Normalized UVVis spectra and (d) fluorescence spectra of P1/345, P1/200, P1/100, P1/ 50, AM-Rh, and $\mathrm{NH}_{2}-\mathrm{Rh}$ in powder form ( $\lambda_{\text {ex }}=530 \mathrm{~nm}$; slit width: 3, 1.5).

Fig. 4a. The low QYs of AM-Rh and $\mathbf{N H}_{\mathbf{2}}-\mathbf{R h}$ are due to aggregation-caused quenching (ACQ). ${ }^{46-48}$

The high QYs of poly(AM-Rh $\mathbf{A}_{\boldsymbol{x}}$ co NIPAM $\left._{\boldsymbol{y}}\right)$ are due to the dispersal effect of the polymer chains preventing molecular aggregation. The fluorescence QYs for the four poly $\left(\mathbf{A M}-\mathbf{R h}_{\boldsymbol{x}}\right.$ co NIPAM $_{y}$ )s (that is, P1/345, P1/200, P1/100, P1/50) decrease as the AM-Rh/NIPAM ratio decreases, which further indicates that ACQ could be effectively prevented by adjusting the proportion of dyes grafted into the polymer. This also means that grafting of ACQ molecules into polymers at appropriate proportions is an effective way to achieve their solid-state luminescence (Fig. 4b).

\section{Conclusion}

In summary, the properties of a hydrochromic material in terms of water solubility and adjustable fluorescence in both solution and solid state are successfully improved by grafting it to a thermosensitive water-soluble polymer. Four poly $\left(\mathbf{A M}-\mathbf{R h}_{\boldsymbol{x}}\right.$ co NIPAM $_{y}$ )s with different dye grafting ratios were obtained by changing the raw ratio of copolymerization. To address the insufficiency of commonly used characterization methods for dye-grafted copolymers, in this work, we put forward a more effective method via UV-Vis absorption spectroscopy and provide detailed methods and steps for calculating dye-grafting ratio $(x / y)$ by spectroscopy combined with GPC technologies. The four copolymers have good solubility in many common organic solvents and even in pure water, meanwhile preserving the hydrochromism of the dye monomer. It was found that the difference in grafting ratio not only influences the maximum absorbance of the colour, but also affects the sensitivity of their hydrochromism. Based on aggregation and dispersion of the four poly(AM-Rh ${ }_{x} \operatorname{co}$ NIPAM $\left._{y}\right)$ s as adjusted by solvent media and temperature, the reversible regulation of their fluorescence properties was successfully realized. In addition, their luminescence in solid state was enabled by this covalent grafting strategy, which effectively overcomes the aggregation-induced fluorescence quenching of dyes. These studies are of great significance for expanding the application of hydrochromic dye molecules in biological fields and promoting green industrialization.

\section{Experimental section}

\section{Materials}

3-Nitrophthalic anhydride (98\%), N,N-diethyl-3-aminophenol (98\%), Pd/C (10\%), o-phthalic anhydride (98\%), and acryloyl chloride (97\%) were purchased from Energy Chemical (Shanghai, China). Tert-butyl alcohol was purchased from East China Reagent (Tianjin, China). Azodiisobutyronitrile (AIBN) was purchased from Aladdin (Shanghai, China). Methanol (99.9\%) and $N, N$-dimethyl formamide (DMF) (99.9\%) were purchased from Yuwang Reagent. Dialysis membrane was purchased from MYM Biological Technology Company Limited. Unless otherwise noted, all the other materials were purchased from Sinopharm Chemical Reagent Beijing Co. without further purification. Deionized water was purified by Milli-Q system.

\section{Instruments}

Absorption spectra were measured using a Shimadzu UV-2550 PC double-beam spectrophotometer. Absorption spectra of solids were measured via the reflective mode of the integrating sphere on an Analytik Jena Specord ${ }^{\circledR} 210$ plus UV/Vis spectrophotometer, using $\mathrm{BaSO}_{4}$ as background and path length of $1 \mathrm{~cm}$. The fluorescence quantum yields (QYs) were measured on a FLS 920 lifetime and steady-state spectrometer. Steady-state fluorescence spectra were measured using a Shimadzu RF5301 PC spectrophotometer. ${ }^{1} \mathrm{H}$ NMR (500 MHz) and ${ }^{13} \mathrm{C}$ NMR $(126 \mathrm{MHz})$ spectra were recorded on a Bruker AVANCE 500 at room temperature. LC-HRMS analysis was performed on an Agilent 1290-Micro TOF-Q II mass spectrometer. $M_{\mathrm{n}}$ and $M_{\mathrm{w}} / M_{\mathrm{n}}$ $(\nexists)$ of poly(AM-Rh $\mathbf{x}_{\boldsymbol{x}}$ co NIPAM $_{\boldsymbol{y}}$ )s were measured using PL-GPC 220 high-temperature gel permeation chromatography (GPC) with Agilent PLgel MIXED-B $(300 \times 7.5 \mathrm{~mm}, 10 \mu \mathrm{m})$, taking

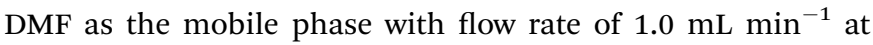
$80{ }^{\circ} \mathrm{C}$, and using RI as the detector and polystyrene (PS) as calibration standard.

\section{Synthesis/preparation of $\mathrm{NH}_{2}-\mathrm{Rh}, \mathrm{AM}-\mathrm{Rh}$ and Poly(AM-Rh ${ }_{x}$ co $\left.\operatorname{NIPAM}_{\boldsymbol{y}}\right) \mathbf{s}$}

$\mathbf{N H}_{2}-\mathbf{R h}$ was prepared according to reported literature. ${ }^{\mathbf{4 9}}$

Synthesis of AM-Rh. $\mathbf{N H}_{2}-\mathbf{R h}(1 \mathrm{mmol}, 457 \mathrm{mg})$ was dissolved in $\mathrm{CH}_{2} \mathrm{Cl}_{2}(10 \mathrm{~mL})$, and $\mathrm{Et}_{3} \mathrm{~N}(2 \mathrm{mmol}, 270 \mu \mathrm{L})$ and acryloyl chloride $(1.2 \mathrm{mmol}, 98 \mu \mathrm{L})$ were added dropwise to the solution 
at $273 \mathrm{~K}$ under dry $\mathrm{N}_{2}$ and stirred at $343 \mathrm{~K}$ for $10 \mathrm{~h}$. The resulting solution was washed with $1 \mathrm{M} \mathrm{HCl}$ and saturated $\mathrm{NaHCO}_{3}$ solutions, dried over $\mathrm{Na}_{2} \mathrm{SO}_{4}$, and concentrated by evaporation. The product was purified by column chromatography (silica, petroleum ether : ethyl acetate : methanol is $5: 5: 1$ ), yielding a bright red solid powder (270 mg, 52\%). ${ }^{1} \mathrm{H}$ NMR $(500 \mathrm{MHz}$, DMSO) $\delta 10.23(\mathrm{~s}, 1 \mathrm{H}), 8.44(\mathrm{~d}, J=8.2 \mathrm{~Hz}, 1 \mathrm{H}), 7.73(\mathrm{t}, J=7.9 \mathrm{~Hz}$, $1 \mathrm{H}), 6.92(\mathrm{~d}, J=7.5 \mathrm{~Hz}, 1 \mathrm{H}), 6.63-6.55(\mathrm{~m}, 3 \mathrm{H}), 6.48-6.44(\mathrm{~m}$, $4 \mathrm{H}), 6.37$ (d, $J=17.0 \mathrm{~Hz}, 1 \mathrm{H}), 5.92$ (d, $J=10.4 \mathrm{~Hz}, 1 \mathrm{H}), 3.37$ (q, $J$ $=6.8 \mathrm{~Hz}, 8 \mathrm{H}), 1.11(\mathrm{t}, J=7.0 \mathrm{~Hz}, 12 \mathrm{H})$. LC-HRMS: $m / z$ calculated for $[\mathrm{M}+\mathrm{H}]^{+}$512.2544, found 512.2536.

Synthesis of P1/50. AM-Rh $(0.1 \mathrm{mmol}, 51 \mathrm{mg})$ and $N$-isopropylacrylamide (NIPAM, $5 \mathrm{mmol}, 565 \mathrm{mg}$ ) were dissolved in tertiary butanol $(t-\mathrm{BuOH})(5 \mathrm{~mL})$ under the protection of $\mathrm{N}_{2}$. The solution was cooled by liquid nitrogen, azodiisobutyronitrile (AIBN) (6 mg, 1\%) was added, and the mixture was stirred at 343 $\mathrm{K}$ for $12 \mathrm{~h}$ under $\mathrm{N}_{2}$. The polymer was concentrated by evaporation and purified by dialysing with methanol in a dialysis bag with a cutoff of 3500 for $60 \mathrm{~h}$. The polymer formed was precipitated with methanol $(3 \mathrm{~mL})$ and diethyl ether $(200 \mathrm{~mL})$. After drying in vacuo, P1/50 was obtained as a dark pink solid (540 mg, 90\%). ${ }^{1} \mathrm{H}$ NMR (500 MHz, $\left.\mathrm{CDCl}_{3}\right) \delta(\mathrm{ppm}) 3.99(\mathrm{~s}, 1 \mathrm{H})$, $3.15(\mathrm{~s}, 0.5 \mathrm{H}), 2.47-1.47(\mathrm{~m}, 3 \mathrm{H}), 1.12(\mathrm{~s}, 6 \mathrm{H})$.

Synthesis of P1/100. AM-Rh (0.1 mmol, $51 \mathrm{mg})$ and NIPAM (10 mmol, $1.2 \mathrm{~g}$ ) were dissolved in $t$-BuOH $(8 \mathrm{~mL})$ under the protection of $\mathrm{N}_{2}$. The solution was cooled by liquid nitrogen, then AIBN (12 mg, 1\%) was added and the mixture stirred at 343 $\mathrm{K}$ for $12 \mathrm{~h}$ under $\mathrm{N}_{2}$. The purification process is the same as that for P1/50. P1/100 was obtained as a pink solid (1.06 g, 89\%).

Synthesis of P1/200. AM-Rh (0.05 mmol, $25 \mathrm{mg})$ and NIPAM $(10 \mathrm{mmol}, 1.2 \mathrm{~g}$ ) were dissolved in $t$-BuOH $(6 \mathrm{~mL})$ under the protection of $\mathrm{N}_{2}$. The solution was cooled by liquid nitrogen, then AIBN (12 mg, 1\%) was added and the mixture stirred at 343 $\mathrm{K}$ for $12 \mathrm{~h}$ under $\mathrm{N}_{2}$. The purification process is the same as that for P1/50. P1/200 was obtained as a pink solid (1.10 g, 92\%).

Synthesis of P1/345. AM-Rh $(0.04 \mathrm{mmol}, 20 \mathrm{mg})$ and NIPAM (13.2 mmol, $1.5 \mathrm{~g}$ ) were dissolved in $t$-BuOH $(9 \mathrm{~mL})$ under the protection of $\mathrm{N}_{2}$. The solution was cooled by liquid nitrogen, then AIBN (16 mg, 1\%) was added and the mixture stirred at 343 $\mathrm{K}$ for $12 \mathrm{~h}$ under $\mathrm{N}_{2}$. The purification process is the same as P1/ 50. P1/345 was obtained as a light pink solid (1.31 g, 87\%).

Synthesis of PO. NIPAM ( $8.8 \mathrm{mmol}, 1.0 \mathrm{~g})$ was dissolved in $t$ $\mathrm{BuOH}(6 \mathrm{~mL})$ under the protection of $\mathrm{N}_{2}$. The solution was cooled by liquid nitrogen, then AIBN (10 mg, 1\%) was added and stirred at $343 \mathrm{~K}$ for $12 \mathrm{~h}$ under $\mathrm{N}_{2}$. The purification process is the same as P1/50. P0 was obtained as a white solid $(0.88 \mathrm{~g}$, $88 \%)$.

\section{Conflicts of interest}

There are no conflicts to declare.

\section{Acknowledgements}

We thank the Natural Science Foundation of China (No. 51603085 and 21572079) for financial support.

\section{References}

1 P. M. Wiggins, Microbiol. Rev., 1990, 54, 432.

2 A. N. Lukyanov and V. P. Torchilin, Adv. Drug Delivery Rev., 2004, 56, 1273.

3 A. J. Humberstone and W. N. Charman, Adv. Drug Delivery Rev., 1997, 25, 103.

4 V. P. Sant, D. Smith and J. C. Leroux, J. Controlled Release, 2004, 97, 301.

5 F. N. Castellano and J. R. Lakowicz, Photochem. Photobiol., 1998, 67, 179.

6 M. Y. Wu, K. Li, C. Y. Li, J. T. Hou and X. Q. Yu, Chem. Commun., 2014, 50, 183.

7 X. Feng, L. Liu, S. Wang and D. Zhu, Chem. Soc. Rev., 2010, 39, 2411.

8 L. Sheng, M. Li, S. Zhu, H. Li, G. Xi, Y. G. Li, Y. Wang, Q. Li, S. Liang, K. Zhong and S. X.-A. Zhang, Nat. Commun., 2014, 5, 3044.

9 D. H. Park, W. Jeong, M. Seo, B. J. Park and J. M. Kim, Adv. Funct. Mater., 2016, 26, 498.

10 T. Maeda and F. Würthner, Chem. Commun., 2015, 51, 7661. 11 M. Pyo, J. Lee, W. Baek, C. W. Lee, B. J. Park and J.-M. Kim, Chem. Commun., 2015, 51, 3177.

12 Z. Wang, J. Zhang, J. Xie, C. Li, Y. Li, S. Liang, Z. Tian, T. Wang, H. Zhang, H. Li, W. Xu and B. Yang, Adv. Funct. Mater., 2010, 20, 3784.

13 M. M. Hawkeye and M. J. Brett, Adv. Funct. Mater., 2011, 21, 3652.

14 D.-H. Park, B. J. Park and J.-M. Kim, Acc. Chem. Res., 2016, 49, 1211.

15 V. K. Singh, R. K. Chitumalla, S. K. Ravi, Y. Zhang, Y. Xi, V. Sanjairaj, C. Zhang, J. Jang and S. C. Tan, ACS Appl. Mater. Interfaces, 2017, 9, 33071.

16 Q. Lou, S. Qu, P. Jing, W. Ji, D. Li, J. Cao, H. Zhang, L. Liu, J. Zhao and D. Shen, Adv. Mater., 2015, 27, 1389.

17 X. Zhang, D. Li, D. Zhou, P. Jing, W. Ji, D. Shen and S. Qu, RSC Adv., 2016, 6, 79620.

18 K. Kolmakov, V. N. Belov, J. Bierwagen, C. Ringemann, V. Müller, C. Eggeling and S. W. Hell, Chem.-Eur. J., 2010, 16, 158.

19 T. Nguyen and M. B. Francis, Org. Lett., 2003, 5, 3245.

20 M. H. Lee, T. V. Giap, S. H. Kim, Y. H. Lee, C. Kang and J. S. Kim, Chem. Commun., 2010, 46, 1407.

21 Y. Xiang, A. Tong, P. Jin and Y. Ju, Org. Lett., 2006, 8(13), 2863.

22 Y. K. Yang, K. J. Yook and J. Tae, J. Am. Chem. Soc., 2005, 127, 16760.

23 G. Xi, L. Sheng, I. Zhang, J. Du, T. Zhang, Q. Chen, G. Li, Y. Zhang, Y. Song, J. Li, Y.-M. Zhang and S.-X. Zhang, ACS Appl. Mater. Interfaces, 2017, 9, 38032.

24 D. Schmaljohann, Adv. Drug Delivery Rev., 2006, 58, 1655. 25 Y. Pan, H. Bao, N. G. Sahoo, T. Wu and L. Li, Adv. Funct. Mater., 2011, 21, 2754.

26 S. Mura, J. Nicolas and P. Couvreur, Nat. Mater., 2013, 12, 991. 
27 A. O. Elzoghby, W. M. Samy and N. A. Elgindy, J. Controlled Release, 2012, 157, 168.

28 J. Guo, W. Yang, Y. Deng, C. Wang and S. Fu, Small, 2005, 1, 737.

29 S. Nayak and L. A. Lyon, Angew. Chem., Int. Ed., 2005, 44, 7686.

30 M. Karg, I. Pastoriza-Santos, J. Pérez-Juste, T. Hellweg and L. M. Liz-Marzán, Small, 2007, 3, 1222.

31 X. Zhang, C. L. Pint, M. H. Lee, B. E. Schubert, A. Jamshidi, K. Takei, H. Ko, A. Gillies, R. Bardhan, J. J. Urban, M. Wu, R. Fearing and A. Javey, Nano Lett., 2011, 11, 3239.

32 J. M. Weissman, H. B. Sunkara, A. S. Tse and S. A. Asher, Science, 1996, 274, 959.

33 M. Zeltner, A. Schätz, M. L. Hefti and W. J. Stark, J. Mater. Chem., 2011, 21, 2991.

34 Y. Z. You, C. Y. Hong, C. Y. Pan and P. H. Wang, Adv. Mater., 2004, 16, 1953.

35 Y. Z. You, K. K. Kalebaila, S. L. Brock and D. Oupicky, Chem. Mater., 2008, 20, 3354.

36 Y. Zhang, S. Furyk, L. B. Sagle, Y. Cho, D. E. Bergbreiter and P. S. Cremer, J. Phys. Chem. C, 2007, 111, 8916.

37 C. W. Scales, A. J. Convertine and C. L. McCormick, Biomacromolecules, 2006, 7, 1389.

38 W. Birnbaum and D. Kuckling, Polym. Chem., 2012, 3, 2039.
39 C.-Y. Hsu, S.-C. Chang, K.-Y. Hsu and Y.-L. Liu, Macromol. Rapid Commun., 2013, 34, 689.

40 J. Hofkens, J. Hotta, K. Sasaki and H. Masuhara, J. Am. Chem. Soc., 1997, 119, 2741.

41 J. Duhamel, Langmuir, 2012, 28, 6527.

42 L. Tang, J. K. Jin, A. Qin, W. Z. Yuan, Y. Mao, J. Mei, J. Z. Sun and B. Z. Tang, Chem. Commun., 2009, 4974.

43 C. Reichardt, Chem. Rev., 1994, 94, 2319.

44 G. Huanga, J. Gaoa, Z. Hua, J. V. St. Johnb, B. C. Ponderb and D. Moro, J. Controlled Release, 2004, 94, 303.

45 Y. Shiraishi, S. Sumiya, K. Manabe and T. Hirai, ACS Appl. Mater. Interfaces, 2011, 3, 4649.

46 R. H. Friend, R. W. Gymer, A. B. Holmes, J. H. Burroughes, R. N. Marks, C. Taliani, D. D. C. Bradley, D. A. Dos Santos, J. L. Brê̂A das, M. Lò̀ gdlund and W. R. Salaneck, Nature, 1999, 397, 121.

47 G. Qian, Z. Zhong, M. Luo, D. Yu, Z. Zhang, Z. Y. Wang and D. Ma, Adv. Mater., 2009, 21, 111.

48 P.-I. Shih, C.-L. Chiang, A. K. Dixit, C.-K. Chen, M.-C. Yuan, R.-Y. Lee, C.-T. Chen, E. W.-G. Diau and C.-F. Shu, Org. Lett., 2006, 8, 2799.

49 I. Zhang, Y. Wang, C. Wan, Z. Xing, W. Li, M. Li and S. X.-A. Zhang, RSC Adv., 2015, 5, 66416. 